\title{
Gravity dual of spatially modulated phase
}

\author{
Shin Nakamura, ${ }^{1}$ Hirosi Ooguri, ${ }^{2,3}$ and Chang-Soon Park ${ }^{2}$ \\ ${ }^{1}$ Department of Physics, Kyoto University, Kyoto 606-8502, Japan \\ ${ }^{2}$ California Institute of Technology, Pasadena, California 91125, USA \\ ${ }^{3}$ IPMU, University of Tokyo, Kashiwa 277-8586, Japan \\ (Received 26 November 2009; published 10 February 2010)
}

\begin{abstract}
We show that the five-dimensional Maxwell theory with the Chern-Simons term is tachyonic in the presence of a constant electric field. When coupled to gravity, a sufficiently large Chern-Simons coupling causes instability of the Reissner-Nordström black holes in anti-de Sitter space. The instability happens only at nonvanishing momenta, suggesting a spatially modulated phase in the holographically dual quantum field theory in $(3+1)$ dimensions, with spontaneous current generation in a helical configuration. The three-charge extremal black hole in the type IIB superstring theory on $\operatorname{AdS}_{5} \times S^{5}$ barely satisfies the stability condition.
\end{abstract}

DOI: 10.1103/PhysRevD.81.044018

PACS numbers: $11.25 . \mathrm{Tq}$

\section{INTRODUCTION}

Instability of black holes in anti-de Sitter space has attracted much attention recently due to its relevance to quantum phase transitions in dual strongly interacting quantum field theory at finite density [1,2]. In this paper, we point out a new type of instability caused by the ChernSimons term. A novel feature is that the instability happens only at nonvanishing momenta, suggesting a spatially modulated phase transition in the holographically dual field theory.

In three dimensions, the Maxwell theory becomes massive when the Chern-Simons term is included [3,4]. In higher dimensions, the Chern-Simons term starts with a higher power in gauge fields, but it can contribute to quadratic fluctuations if there is a nonzero background gauge field. In this paper, we will show that the Maxwell theory in five dimensions with the Chern-Simons term becomes tachyonic if we turn on a constant electric field. In contrast, a background magnetic field does not cause instability, but it makes the gauge field massive as in three dimensions. ${ }^{1}$

Chern-Simons terms abound in supergravity theories, and charged black hole solutions in these theories provide an interesting laboratory in which to study the instability and its implications since these solutions carry background electric fields. The near-horizon geometry of the fivedimensional extremal Reissner-Nordström black hole in $\mathrm{AdS}_{5}$ is $\mathrm{AdS}_{2} \times \mathbb{R}^{3}$ with the gauge field strength propor-

\footnotetext{
${ }^{1}$ To our knowledge, [5] is the first paper to point out that the Chern-Simons term in five dimensions induces instability. They considered a system consisting of two non-Abelian gauge fields coupled to an adjoint scalar field with a tachyonic mass as a holographic model of QCD and reduced it to four dimensions before studying its spectrum. Though their setup and analysis are different from ours, the dispersion relation we derive in Sec. II is related to theirs. We will point this out at an appropriate place in Sec. II.
}

tional to the volume form of $\mathrm{AdS}_{2}$. The background electric field causes mixing of the gauge field with the metric at the quadratic order, and we will take it into account in our stability analysis. We find a critical value $\alpha_{\text {crit }}$ of the Chern-Simons coupling $\alpha$ above which the near-horizon geometry becomes unstable for some range of momenta $k$ in $\mathbb{R}^{3}$. Interestingly, the range excludes $k=0$, i.e. the instability happens only at nonzero spatial momentum.

The Reissner-Nordström black hole solution in $\mathrm{AdS}_{5}$ gives a holographic description of a thermodynamic state in the dual conformal field theory at finite temperature $T$ and chemical potential $\mu{ }^{2}$. We find that, for $\alpha>\alpha_{\text {crit }}$, there is a critical temperature $T_{\mathrm{c}}(\alpha)$ below which the black hole solution becomes unstable, as shown in Fig. 1. The instability happens at a range of momenta, which becomes wider as $T$ is lowered but never includes $k=0$, as shown in Fig. 2. We find an interesting subtlety in the zero temperature limit; the unstable range is wider than the range expected from the analysis near the horizon of the extremal black hole. It turns out that the near-horizon analysis gives a sufficient but not necessary condition since there are unstable modes in the full Reissner-Nordström solution which do not reduce to normalizable modes in $\operatorname{AdS}_{2} \times$ $\mathbb{R}^{3}$ in the near-horizon limit.

In the dual field theory in $(3+1)$ dimensions, the instability of the Reissner-Nordström solution can be interpreted as a signal of a novel phase transition at finite chemical potential where the charge current $\vec{J}(x)$ dual to the gauge field develops a position dependent expectation value of the form

$$
\langle\vec{J}(x)\rangle=\operatorname{Re}\left(\vec{u} e^{i k x}\right),
$$

with nonzero momentum $k$. The constant vector $\vec{u}$ is circularly polarized as

\footnotetext{
${ }^{2}$ References [6,7] studied the thermodynamic properties of the Reissner-Nordström AdS black hole. Its relation to Fermi liquid is discussed in [8].
} 


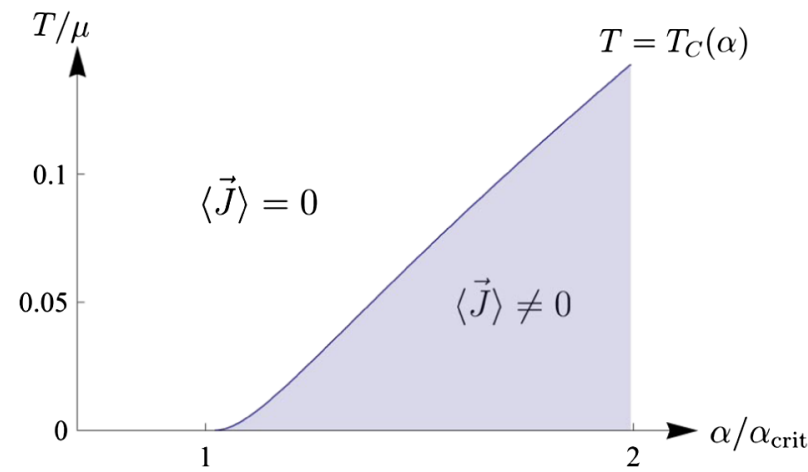

FIG. 1 (color online). Critical temperature as a function of the Chern-Simons coupling $\alpha$. The shaded region indicates a phase with a nonzero expectation value of the conserved current $\vec{J}$ which is helical and position dependent.

$$
\vec{k} \times \vec{u}= \pm i|k| \vec{u}
$$

where the sign is correlated to the sign of the ChernSimons couping as we will explain later. The vacuum expectation value (1.1) is helical and breaks translational and rotational symmetries in three spatial dimensions, while preserving a certain combination of the two. The configuration reminds us of the cholesteric phase of liquid crystals.

Spatially modulated phases are known in condensed matter physics and in QCD. In the Fulde-Ferrell-LarkinOvchinnikov phase, a Cooper pair of two species of fermions with different Fermi momenta condenses with nonvanishing total momentum $[9,10]$. An analogous effect in QCD was studied in [11]. It has also been shown that finite density QCD in the large $N_{c}$ limit is unstable against forming the chiral density wave $[12,13]$. Gravity theories with Chern-Simons terms may provide dual descriptions of such systems and clarify aspects of their phase transitions.

For example, the Brazovskii model [14] generates a spatially modulated phase, and it has been applied to a variety of physical problems [15]. In this model, a nonstandard dispersion relation is postulated so that the fluc- tuation spectrum has a minimum at nonzero momentum. The gravity theory discussed in this paper provides a holographic realization of a similar dispersion relation.

In this paper, we use the Maxwell theory with the ChernSimons term coupled to the gravity in $\mathrm{AdS}_{5}$ as a phenomenological model of quantum critical phenomena in the spirit of $[1,2]$. To have an explicit description of the field content and interactions of the dual field theory, we need to identify a specific superstring construction where the instability takes place. We examined the simplest case of the three-charge black hole in the type IIB superstring theory on $\mathrm{AdS}_{5} \times S^{5}$ and found that the Chern-Simons coupling of the low energy gravity theory barely satisfies the stability bound. More specifically, when the three charges are the same, the effective Chern-Simons coupling $\alpha$ is only $0.4 \%$ less than the critical value $\alpha_{\text {crit }}$ for the instability. There is a limit of an extreme ratio of charges, where an effective $\alpha$ coincides with $\alpha_{\text {crit }}$ and the black hole becomes marginally stable.

This seems to indicate that, if we survey a wider class of examples, we may be able to find a theory with a ChernSimons coupling large enough to cause an instability. Generally speaking, the Chern-Simons coupling for a gauge field in $\mathrm{AdS}_{5}$ is proportional to the chiral anomaly of the corresponding current in the dual conformal field theory [16]. In particular, for the type IIB superstring theory on $\mathrm{AdS}_{5}$ times a toric Sasaki-Einstein manifold, the Chern-Simons coupling is determined by the toric data, or equivalently by the combinatorial data of the quiver diagram for the dual gauge theory [17]. It would be interesting to find an explicit example where the ChernSimons coupling exceeds the stability bound. Or, one may try to prove that such theories are all in the Swampland $[18,19]$.

Even for a theory with $\alpha<\alpha_{\text {crit }}$, in which the ChernSimons term is not strong enough to cause instability, the nonstandard dispersion relation is noteworthy by itself with potential applications to physical problems. For example, a plasmino in QCD is a collective mode of quarks whose spectrum has a minimum at a nonzero momentum [20,21].
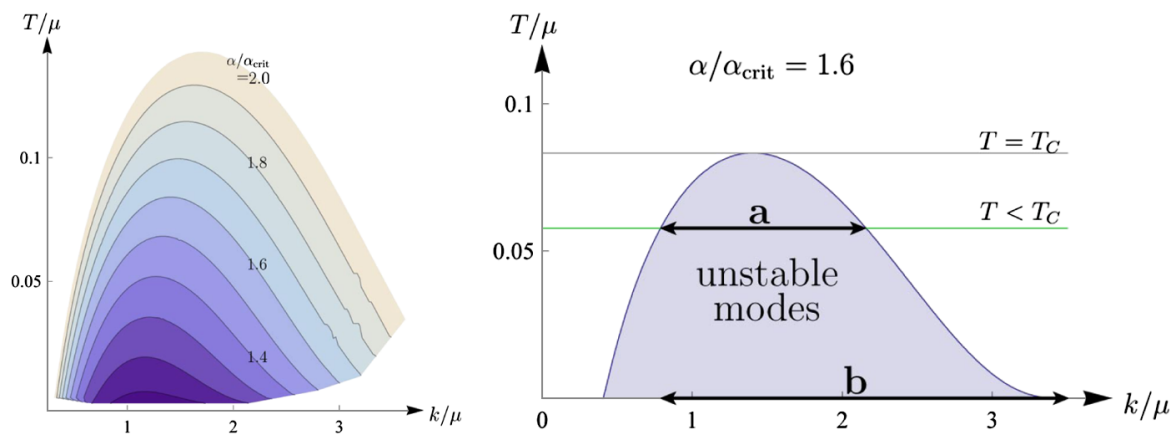

FIG. 2 (color online). The left figure indicates unstable regions for various values of the Chern-Simons coupling $\alpha$. The right figure is for a particular choice of the Chern-Simons coupling $\alpha=1.6 \alpha_{\text {crit }}$. The critical temperature $T_{C}$ is the maximum temperature with unstable modes. The figure indicates the unstable range a for some temperature $T<T_{C}$. The range $\mathbf{b}$ is derived from the near-horizon analysis at $T=0$. Note that the actual range of unstable momenta is wider. 
In the presence of a plasmino, the dilepton production rate diverges at the minimum of the spectrum due to the Van Hove singularity, i.e., the divergence of the density of states per unit energy [22,23].

We should also point out that another type of instability of rotating charged black holes was suggested in [24,25]. While the Chern-Simons term seems to play a role there, we have found no obvious connection to the instability discussed in this paper. Effects of the bulk Chern-Simons terms on hydrodynamics of the dual field theories have been studied in [26-29]. In [30,31], dispersion relations of hydrodynamic waves in the Reissner-Nordström geometry with the Chern-Simons term are discussed. Since the authors of these papers relied on power series expansions around $k=0$, they did not observe the instability we found in this paper since the range of instability is away from $k=$ 0 as shown in Fig. 2.

This paper is organized as follows. In Sec. II, we show that the five-dimensional Maxwell theory with the ChernSimons term is unstable in the presence of a constant electric field. The metric is treated as nondynamical in this analysis. In Sec. III, we turn on the metric fluctuation and study the stability of the near-horizon geometry of the extremal Reissner-Nordström black hole in $\mathrm{AdS}_{5}$. In Sec. IV, we generalize the analysis of Sec. III to the full Reissner-Nordström solution. We solve the linearized equations around the black hole geometry and identify the critical temperature $T_{\text {crit }}$ of the phase transition. We examine the onset of the phase transition and interpret the result from the point of view of the dual field theory. In Sec. V, we show that the three-charge black hole in thetype IIB superstring theory on $\operatorname{AdS}_{5} \times S^{5}$ is barely stable against the type of instability discussed in this paper.

\section{MAXWELL THEORY WITH CHERN-SIMONS TERM}

It is well-known that the three-dimensional Maxwell theory with the Chern-Simons term is massive $[3,4]$. The equation of motion for the 2 -form field strength $F$ is given by

$$
d^{*} F+\alpha F=0,
$$

where $\alpha$ is the Chern-Simons coupling constant. Applying $d^{*}$ to this equation and using the Bianchi identity $d F=0$, one finds

$$
\square F=d^{*} d^{*} F=-\alpha d^{*} F=\alpha^{2} F .
$$

Thus, the Chern-Simons term in three dimensions induces the mass $|\alpha|$ of the gauge field.

Surprisingly, we find that the Chern-Simons term in five dimensions can turn the Maxwell theory tachyonic. In this section, we will demonstrate this by treating gravity as nondynamical. Coupling to gravity will be studied in the following sections. Consider the following Lagrangian density:

$$
\mathcal{L}=-\frac{1}{4} \sqrt{-g} F_{I J} F^{I J}+\frac{\alpha}{3 !} \epsilon^{I J K L M} A_{I} F_{J K} F_{L M},
$$

with the equation of motion

$$
\partial_{J}\left(\sqrt{-g} F^{J I}\right)+\frac{\alpha}{2} \epsilon^{I J K L M} F_{J K} F_{L M}=0 .
$$

We use the almost positive convention for the metric $g_{I J}(I$, $J=0, \ldots, 4)$. Choose a background solution $F^{(0)}$ and linearize (2.3) around it by substituting $F=F^{(0)}+f$ in (2.3). The linearized equation for $f$ is given by

$$
\partial_{J}\left(\sqrt{-g} f^{J I}\right)+\alpha \epsilon^{I J K L M} F_{J K}^{(0)} f_{L M}=0 .
$$

If $F^{(0)}$ is magnetic, this equation is similar to (2.1); the fluctuation $f_{I J}$ is massive and the configuration is stable. If $F^{(0)}$ is electric, on the other hand, (2.4) has tachyonic modes as we now explain.

Suppose the five-dimensional space is flat $\mathbb{R}^{1,4}$, regard it as the product $\mathbb{R}^{1,1} \times \mathbb{R}^{3}$, and use coordinates $\left(x^{\mu=0,1}, y^{i=2,3,4}\right)$. Let us turn on a constant electric field in the $x^{1}$ direction,

$$
F_{\mu \nu}^{(0)}=E \epsilon_{\mu \nu}, \quad F_{\mu i}^{(0)}=0, \quad F_{i j}^{(0)}=0 .
$$

The equation of motion (2.4) is then

$$
\begin{aligned}
\partial^{\mu} f_{\mu \nu}+\partial^{i} f_{i \nu} & =0, \\
\partial^{\mu} f_{\mu i}+\partial^{j} f_{j i}-2 \alpha E \epsilon_{i j k} f_{j k} & =0 .
\end{aligned}
$$

Our $\epsilon$-symbol convention is such that $\epsilon_{01}=1$ and $\epsilon_{234}=$ 1. By multiplying $\epsilon_{i j k} \partial_{j}$ to the second equation, we obtain

$$
\left(\partial^{\mu} \partial_{\mu}+\partial^{j} \partial_{j}\right) f_{i}-4 \alpha E \epsilon_{i j k} \partial_{j} f_{k}=0,
$$

where

$$
f_{i}=\frac{1}{2} \epsilon_{i j k} f_{j k} .
$$

To derive (2.7), we used the Bianchi identities

$$
\partial_{i} f_{\mu j}-\partial_{j} f_{\mu i}=\partial_{\mu} f_{i j}, \quad \epsilon_{i j k} \partial_{i} f_{j k}=2 \partial^{i} f_{i}=0 .
$$

In the momentum basis $e^{i p_{\mu} x^{\mu}+i k_{i} y^{i}}$, the operator $\epsilon_{i j k} \partial_{j}$ has eigenvalues $\pm k$ and 0 , where $k=|\vec{k}|$. However, the eigenvalue 0 corresponds to $f_{i} \sim k_{i}$, which is prohibited by the Bianchi identity $k^{i} f_{i}=0$. Thus, the linearized Eq. (2.7) gives the dispersion relation ${ }^{3}$

$$
\left(p_{0}\right)^{2}-\left(p_{1}\right)^{2}=k^{2} \pm 4 \alpha E k=(k \pm 2 \alpha E)^{2}-4 \alpha^{2} E^{2} .
$$

We find tachyonic modes in $\mathbb{R}^{1,1}$ in the range of $0<k<$ $4|\alpha E|$.

It is instructive to compare this with the case when we turn on a constant magnetic field,

\footnotetext{
${ }^{3}$ At this point, we should note that there is a similarity of this dispersion relation to Eq. (17) of [5] if we set $m_{\rho}=m_{a_{1}}$ in the paper and interpret $m_{\rho}^{2}$ as being equal to $\left(p_{1}\right)^{2}$.
} 


$$
F_{34}^{(0)}=-F_{43}^{(0)}=B, \quad F_{I J}^{(0)}=0 \quad \text { (otherwise). }
$$

By repeating the previous analysis, we find the dispersion relation,

$$
\begin{aligned}
\left(p_{0}\right)^{2}-\left(p_{1}\right)^{2}-\left(k_{2}\right)^{2}= & \left(\sqrt{\left(k_{3}\right)^{2}+\left(k_{4}\right)^{2}+4 \alpha^{2} B^{2}}\right. \\
& +2|\alpha B|)^{2} .
\end{aligned}
$$

In particular, when $k_{3}=k_{4}=0$, the equation gives $p_{0}^{2}-$ $p_{1}^{2}-k_{2}^{2}=(4 \alpha B)^{2}$, reproducing the topologically massive gauge field in three dimensions.

In the following sections, we will examine stability of the extremal Reissner-Nordström black hole in $\mathrm{AdS}_{5}$. If the boundary theory is on $\mathbb{R}^{1,3}$, the near-horizon geometry of an extremal black hole takes the form $\mathrm{AdS}_{2} \times \mathbb{R}^{3}$ with an electric field proportional to the volume form of $\mathrm{AdS}_{2}$. In such a configuration, the effective mass squared in $\mathrm{AdS}_{2}$ is again given by the right-hand side of (2.8). The configuration is unstable if $-4 \alpha^{2} E^{2}$ violates the BreitenlohnerFreedman bound $m_{\mathrm{BF}}^{2}$ in $\mathrm{AdS}_{2}$, namely,

$$
4 \alpha^{2} E^{2}>\left|m_{\mathrm{BF}}^{2}\right|=\frac{1}{4 r_{2}^{2}},
$$

where $r_{2}$ is the curvature radius of $\mathrm{AdS}_{2}$. If this inequality is satisfied, the instability happens for nonzero momenta in the range

$$
\begin{aligned}
2|\alpha E|\left(1-\sqrt{1-\frac{1}{16 \alpha^{2} E^{2} r_{2}^{2}}}\right) \\
\quad<k<2|\alpha E|\left(1+\sqrt{1-\frac{1}{16 \alpha^{2} E^{2} r_{2}^{2}}}\right) .
\end{aligned}
$$

It is interesting to note that the zero momentum $k=0$ is excluded from the instability range. Thus, the condensate of the gauge field happens for nonzero momentum in the $\mathbb{R}^{3}$ direction of the near-horizon geometry.

As we shall see in the next section, the value of $\alpha$ for the minimal gauged supergravity is such that $4 \alpha^{2} E^{2}$ exceeds the stability bound as in (2.10). This, however, does not mean that extremal charged black holes in the minimal gauged supergravity are unstable since we must take into account the coupling of the Maxwell field to other degrees of freedom in the supergravity theory. We will perform this analysis in the next section.

\section{COUPLING TO GRAVITY}

The background electric field causes mixing of the gauge field with the metric at the quadratic order, and it modifies the stability condition. In this section, we will study stability of the near-horizon geometry of the extremal Reissner-Nordström solution in $\mathrm{AdS}_{5}$. It is a solution to the Maxwell theory with the Chern-Simons term coupled to the Einstein gravity with negative cosmological constant

$$
\begin{aligned}
16 \pi G_{5} \mathcal{L}= & \sqrt{-g}\left(R+\frac{12}{\ell^{2}}-\frac{1}{4} \ell^{2} F_{I J} F^{I J}\right) \\
& +\frac{\alpha}{3 !} \ell^{3} \epsilon^{I J K L M} A_{I} F_{J K} F_{L M} .
\end{aligned}
$$

The curvature radius $r_{5}$ of the $\mathrm{AdS}_{5}$ solution in this theory is equal to $\ell$. In the following, we will work in the unit of $\ell=1$. This is also the Lagrangian density of the minimal gauged supergravity in five dimensions [32]. In this case, supersymmetry determines the Chern-Simons coupling $\alpha$ as

$$
\alpha=\frac{1}{2 \sqrt{3}} .
$$

In this and the next sections, we will treat (3.1) as a phenomenological Lagrangian with $\alpha$ as its parameter.

$$
\text { A. } \operatorname{AdS}_{2} \times \mathbb{R}^{3}
$$

Let us first consider the extremal black hole solution which is asymptotic to $\mathrm{AdS}_{5}$ in the Poincaré coordinates. It describes the dual conformal field theory on $\mathbb{R}^{1,3}$ with nonzero chemical potential and at zero temperature. The near-horizon geometry of the extremal black hole is $\mathrm{AdS}_{2} \times \mathbb{R}^{3}$ with the metric

$$
d s^{2}=\frac{-\left(d x^{0}\right)^{2}+\left(d x^{1}\right)^{2}}{12\left(x^{1}\right)^{2}}+d \vec{y}^{2}, \quad \vec{y}=\left(y^{2}, y^{3}, y^{4}\right) .
$$

Note that the curvature radius $r_{2}$ of $\mathrm{AdS}_{2}$ is $1 / \sqrt{12}$; the curvature is stronger near the horizon. The electric field strength near the horizon is proportional to the volume form of $\mathrm{AdS}_{2}$ and is given by

$$
F_{01}^{(0)}=\frac{E}{12\left(x^{1}\right)^{2}}, \quad E= \pm 2 \sqrt{6} .
$$

For the minimal gauged supergravity with $\alpha$ given by (3.2),

$$
4 \alpha^{2} E^{2}=8>\left|m_{\mathrm{BF}}^{2}\right|=\frac{1}{4 r_{2}^{2}}=3 \text {. }
$$

Thus, if gravity is treated as nondynamical, the gauge field fluctuation near the horizon violates the BreitenlohnerFreedman bound for this value of $\alpha$.

We decompose the metric $g_{I J}$ into the background $g_{I J}^{(0)}$ and the fluctuation $h_{I J}$ as $g_{I J}=g_{I J}^{(0)}+h_{I J}$. The indices are raised/lowered by using the background metric. Notice that $g^{I J}=g^{I J(0)}-h^{I J}+O\left(h^{2}\right)$ so that $g^{I J} g_{J K}=\delta^{I}{ }_{K}$. In the presence of the background electric field $F_{\mu \nu}^{(0)}$, the unstable gauge field components $f_{\mu i}, f_{i j} \neq 0$ mix with the offdiagonal elements $h_{\mu}^{i}$ of the metric perturbation through the gauge kinetic term

$$
F_{I J} F^{I J}=4 F^{(0) \mu \nu} h_{\mu}{ }^{i} f_{\nu i}+\cdots .
$$

Thus, in the stability analysis, we have to take into account 
the mixing. One can think of $h_{\mu}{ }^{i}$ as the Kaluza-Klein gauge field upon reduction on $\mathbb{R}^{3}$. Since we are considering a sector with nonzero momentum $\vec{k}$ along $\mathbb{R}^{3}$, the KaluzaKlein gauge field on $\mathrm{AdS}_{2}$ has mass $\vec{k}^{2}$.

To examine the stability of the black hole solution, we can apply the standard linear perturbation theory. In the present situation, however, there is a simpler way as we describe here. Suppose that the momentum $\vec{k}$ on $\mathbb{R}^{3}$ is in the $y^{2}$ direction. To derive the effective action for the Kaluza-Klein gauge field $h_{\mu}^{i}$ in $\mathrm{AdS}_{2}$, it is convenient to reduce the Einstein action in (3.1) along the $y^{3,4}$ directions first. This gives rise to two gauge fields $\left(h_{\mu}^{i}, h_{2}^{i}\right)(i=3,4)$ on $\mathrm{AdS}_{2} \times \mathbb{R}_{y^{2}}$, with the effective Lagrangian

$$
\begin{aligned}
\sqrt{-g_{5 d}^{(0)}}(R+12) \rightarrow & \sqrt{-g_{3 d}^{(0)}} \\
& \times\left[-\sum_{i=3,4}\left(\frac{1}{4} K_{\mu \nu}^{i} K^{i \mu \nu}+\frac{1}{2} K_{\mu 2}^{i} K^{i \mu 2}\right)\right. \\
& \left.+\left(\text { terms not involving } h_{\mu}{ }^{i}, h_{2}{ }^{i}\right)\right]
\end{aligned}
$$

where the gauge field strengths are

$$
\begin{gathered}
K_{\mu \nu}^{i}=\partial_{\mu} h_{\nu}{ }^{i}-\partial_{\nu} h_{\mu}{ }^{i}, \quad K_{\mu 2}^{i}=\partial_{\mu} h_{2}{ }^{i}-\partial_{2} h_{\mu}{ }^{i} \\
(\mu, \nu=0,1 ; i=3,4) .
\end{gathered}
$$

Upon further reduction in the $y^{2}$ direction with momentum $k$, the effective Lagrangian density for the Kaluza-Klein gauge field is

$$
\mathcal{L}_{\text {eff }}=-\sqrt{-g_{2 d}^{(0)}} \sum_{i=3,4}\left[\frac{1}{4} K_{\mu \nu}^{i} K^{i \mu \nu}+\frac{1}{2}\left|\partial_{\mu} h_{2}{ }^{i}-i k h_{\mu}{ }^{i}\right|^{2}\right] .
$$

We see that the off-diagonal elements $h_{\mu}{ }^{i}(i=3,4)$ of the metric fluctuation give rise to two massive gauge fields of mass $|k|$ on $\mathrm{AdS}_{2}$ with $h_{2}{ }^{i}$ serving as the requisite Stückelberg fields.

Let us dualize the Kaluza-Klein field strength $K_{\mu \nu}^{i}$ on $\mathrm{AdS}_{2}$ and write it as a function $K_{i}$ times the volume form

$$
K_{01}^{i}=\frac{K_{i}}{12\left(x^{1}\right)^{2}} .
$$

The equations of motion for $f_{i}=\frac{1}{2} \epsilon_{i j k} f_{j k}$ and $K_{j}$ are derived from the Lagrangian density which is (2.2) plus (3.8) with the coupling (3.6). They can be organized into the form

$$
\begin{array}{r}
\left(\square_{\mathrm{AdS}_{2}}+\partial^{j} \partial_{j}\right) f_{i}-4 \alpha E \epsilon_{i j k} \partial_{j} f_{k}+E \epsilon_{i j k} \partial_{j} K_{k}=0, \\
E \square_{\mathrm{AdS}_{2}} f_{i}+\left(\square_{\mathrm{AdS}_{2}}+\partial^{j} \partial_{j}\right) \epsilon_{i j k} \partial_{j} K_{k}=0 .
\end{array}
$$

The effective mass $m$ of these fields in $\mathrm{AdS}_{2}$ can then be computed by solving

$$
\operatorname{det}\left(\begin{array}{cc}
m^{2}-k^{2}-4 \alpha E k & E \\
E m^{2} & m^{2}-k^{2}
\end{array}\right)=0,
$$

where $k= \pm|k|$. We find

$$
\begin{aligned}
m^{2}= & \frac{1}{2}\left(2 k^{2}+E^{2}+4 \alpha E k\right. \\
& \left. \pm \sqrt{E^{4}+8 \alpha E^{3} k+4\left(1+4 \alpha^{2}\right) E^{2} k^{2}}\right) .
\end{aligned}
$$

Minimizing $m^{2}$ with respect to $k$ and choosing the minus sign in (3.11), we obtain the lowest value of $m^{2}$ as

$m_{\min }^{2}=\frac{E^{2}\left(-64 \alpha^{6}-24 \alpha^{4}+6 \alpha^{2}-\left(16 \alpha^{4}+4 \alpha^{2}+1\right)^{3 / 2}+1\right)}{2\left(4 \alpha^{2}+1\right)^{2}}$.

Substituting $E=2 \sqrt{6}$ for the near-horizon geometry, we find numerically that the lowest value of $m^{2}$ violates the Breitenlohner-Freedman bound if

$$
|\alpha|>\alpha_{\text {crit }}=0.2896 \cdots .
$$

The value of $\alpha$ for the minimal gauged supergravity is

$$
\alpha=\frac{1}{2 \sqrt{3}}=0.2887 \cdots .
$$

Thus, the supergravity theory is stable against the fluctuation of the gauge field, but barely so (with a margin less than $0.4 \%$ ).

\section{B. $\operatorname{AdS}_{2} \times S^{3}$}

For completeness, let us consider the case when the boundary theory is on $\mathbb{R} \times S^{3}$. The near-horizon geometry is $\mathrm{AdS}_{2} \times S^{3}$. Let us denote the curvature radii of $\operatorname{AdS}_{2}$ and $S^{3}$ by $r_{2}$ and $r_{3}$, respectively. They are related to the electric field strength $E$ and the cosmological constant $\Lambda$, which is -6 in the $\mathrm{AdS}_{2} \times \mathbb{R}^{3}$ limit, by

$$
\Lambda=-\frac{1}{2 r_{2}^{2}}+\frac{2}{r_{3}^{2}}, \quad E^{2}=\frac{2}{r_{2}^{2}}+\frac{4}{r_{3}^{2}} .
$$

Note that, in the limit $r_{3} \rightarrow \infty$, where $S^{3}$ becomes $\mathbb{R}^{3}$, this reproduces $E= \pm 2 \sqrt{6}$ in our unit.

As in the previous case, we consider fluctuations of the metric $g_{I J}=g_{I J}^{(0)}+h_{I J}$ and the gauge field $F_{I J}=F_{I J}^{(0)}+$ $f_{I J}$ from their classical values indicated by ${ }^{(0)}$. We expand the Einstein equation,

$$
R_{I J}-\frac{1}{2} g_{I J} R=\frac{1}{2}\left(F_{I K} F_{J}{ }^{K}-\frac{1}{4} g_{I J} F_{K L} F^{K L}\right),
$$

and the Maxwell equation modified by the Chern-Simons term

$$
\sqrt{-g} \nabla_{J} F^{J I}+\frac{\alpha}{2} \epsilon^{I J K L M} F_{J K} F_{L M}=0,
$$

to the linear order in $h_{I J}$ and $f_{I J}$.

The linearized equations for $f_{i j}$ and $K_{i}$, where $K_{i}$ is defined such that $K_{i}(\operatorname{vol~AdS})_{\mu \nu}=2 \nabla_{[\mu} h_{\nu] i}$, can be writ- 
ten as

$$
\begin{aligned}
& \left(\square_{\mathrm{AdS}_{2}}+\Delta_{S^{3}}\right) f-4 \alpha E d^{*} f+E d K=0, \\
& E \square_{\mathrm{AdS}_{2}} f+\left(\square_{\mathrm{AdS}_{2}}+\Delta_{S^{3}}+\frac{4}{r_{3}^{2}}\right) d K=0 .
\end{aligned}
$$

These equations are similar to (3.9), except for the last term $\frac{4}{r_{3}^{2}}$ in the second equation. Here ${ }^{*}$ means the Hodge dual on $S^{3}$. Since $d^{*}$ is Hermitian when acting on the space of twoforms on $S^{3}$, decompose $f$ into its eigenstate. Its eigenvalue is known to be $k= \pm(n+2) / r_{3}$, where $n=0,1,2, \ldots$. Since $\Delta_{S^{3}}=-\left(d^{*}\right)^{2}$ when acting on $f$ satisfying the Bianchi identify $d f=0$, we can set $\Delta_{S^{3}}=-k^{2}$.

The mass $m$ on $\mathrm{AdS}_{2}$ then satisfies the determinant equation

$$
\operatorname{det}\left(\begin{array}{cc}
m^{2}-k^{2}-4 \alpha E k & E \\
E m^{2} & m^{2}-k^{2}+4 / r_{3}^{2}
\end{array}\right)=0 .
$$

This can be solved to obtain,

$$
\begin{aligned}
m^{2}= & \frac{1}{2}\left[2 k^{2}+E^{2}+4 \alpha E k-\frac{4}{r_{3}^{2}}\right. \\
& \left. \pm \sqrt{E^{4}+8 \alpha E^{3} k+\frac{16}{r_{3}^{4}}+\frac{32 \alpha k}{r_{3}^{2}}+4 E^{2}\left(k^{2}+4 \alpha^{2} k^{2}-\frac{2}{r_{3}^{2}}\right)}\right]
\end{aligned}
$$

In the limit of $r_{3} \rightarrow \infty$, this reduces to the previous result (3.11).

We have numerically checked that, for a wide range of $\Lambda$ and $E$, the Breitenlohner-Freedman bound is not violated in the minimal gauged supergravity, where $\alpha=\frac{1}{2 \sqrt{3}}$. It is interesting to note that, in the limit of $\Lambda \rightarrow 0$ but with nonzero $E$, the lowest $m^{2}$ in (3.18) saturates the Breitenlohner-Freedman bound [33], which is

$$
-\frac{1}{4 r_{2}^{2}}=-\frac{1}{12}\left(E^{2}-2 \Lambda\right)=-\frac{E^{2}}{12}
$$

\section{PHASE TRANSITION AND CRITICAL TEMPERATURE}

In the last section, we studied the instability of the nearhorizon region of the extremal Reissner-Nordström solution. This gives a sufficient condition for the solution to be unstable. However, as we will see in this section, the condition turns out to be not necessary. To clarify the nature of the phase transition and identify the critical temperature, we study linear perturbation to the full Reissner-Nordström black hole in $\mathrm{AdS}_{5}$.

\section{A. Geometry and equations}

The Reissner-Nordström black hole has the metric

$$
\begin{aligned}
d s^{2} & =-H(r) d t^{2}+\frac{1}{H(r)} d r^{2}+r^{2} d \vec{y}^{2}, \\
\vec{y} & =\left(y^{2}, y^{3}, y^{4}\right) .
\end{aligned}
$$

Note $\sqrt{-g^{(0)}}=r^{3}$. The gauge field strength is given by

$$
F^{(0)}=\frac{Q}{r^{3}} d t \wedge d r
$$

The function $H(r)$ is given by

$$
H(r)=r^{2}\left[1-\left(1+\frac{\mu^{2}}{3 r_{+}^{2}}\right)\left(\frac{r_{+}}{r}\right)^{4}+\frac{\mu^{2}}{3 r_{+}^{2}}\left(\frac{r_{+}}{r}\right)^{6}\right],
$$

where $Q=-2 \mu r_{+}^{2}$.

The equation of motion coming from the variation of the gauge field $a_{i}$ is

$$
\begin{gathered}
\partial_{\mu}\left(\sqrt{-g^{(0)}} f^{\mu i}\right)+\partial_{j}\left(\sqrt{-g^{(0)}} f^{j i}\right)-2 \alpha \frac{Q}{r^{3}} \epsilon_{i j k} f_{j k} \\
-\partial_{\rho}\left(\sqrt{-g^{(0)}} \frac{Q}{r^{3}} \epsilon^{\mu \rho} h_{\mu}{ }^{i}\right)=0 .
\end{gathered}
$$

In the black hole background (4.1), it becomes

$$
\begin{gathered}
-\frac{r}{H(r)} \partial_{t} f_{t i}+\partial_{r}\left(r H(r) f_{r i}\right)+\frac{1}{r} \partial_{j} f_{j i}-2 \alpha \frac{Q}{r^{3}} \epsilon_{i j k} f_{j k} \\
+Q K^{i}=0
\end{gathered}
$$

where $K^{i}=\partial_{t} h_{r}{ }^{i}-\partial_{r} h_{t}{ }^{i}$. By operating $\epsilon_{i j k} \partial_{j}$ on this equation, we obtain

$$
\begin{gathered}
-\frac{r}{H(r)} \partial_{t}^{2} f_{i}+\partial_{r}\left(H(r) r \partial_{r} f_{i}\right)+\frac{1}{r} \Delta_{\mathbb{R}^{3}} f_{i} \\
-4 \alpha \frac{Q}{r^{3}} \epsilon_{i j k} \partial_{j} f_{k}+Q \epsilon_{i j k} \partial_{j} K^{k}=0,
\end{gathered}
$$

where $f_{i}=\frac{1}{2} \epsilon_{i j k} f_{j k}$ and $\Delta_{\mathbb{R}^{3}}=\partial_{y^{2}}^{2}+\partial_{y^{3}}^{2}+\partial_{y^{4}}^{2}$.

To obtain the equation of motion that comes from the variation of the off-diagonal metric elements, let us use the Kaluza-Klein reduction in the presence of momentum $\vec{k}$ along the $y^{2}$ direction. The effective Lagrangian has the form

$$
\begin{aligned}
\mathcal{L}_{\mathrm{eff}}= & -r^{3} \sqrt{-g_{2 d}^{(0)}} \sum_{i=3,4}\left[\frac{1}{4} r^{2} K_{\mu \nu}^{i} K^{i \mu \nu}\right. \\
& \left.+\frac{1}{2}\left|\partial_{\mu} h_{2}{ }^{i}-i k h_{\mu}{ }^{i}\right|^{2}\right] .
\end{aligned}
$$

The $r^{3}$ factor comes from the volume form on the $\mathbb{R}^{3}$ directions with coordinates $\vec{y}$. The equation of motion coming from the variation with respect to the metric is given by 


$$
\partial_{\nu}\left(r^{5} K^{\nu \mu i}\right)+r^{3}(-i k)\left(\partial^{\mu} h_{2}{ }^{i}-i k h^{\mu i}\right)-Q \epsilon^{\mu \rho} f_{\rho i}=0 .
$$

Acting on the operator $\epsilon_{\alpha \mu} \partial_{\beta} g^{\alpha \beta(0)} \frac{1}{r^{3}}$, we can eliminate the term containing $\partial^{\mu} h_{2}^{i}$. Using $\epsilon_{\alpha \mu} \partial_{\nu}+\epsilon_{\mu \nu} \partial_{\alpha}+$ $\epsilon_{\nu \alpha} \partial_{\mu}=0$ in two dimensions, we obtain

$$
\begin{gathered}
-\frac{1}{2} \epsilon_{\mu \nu} \partial_{\beta} g^{\alpha \beta(0)} \frac{1}{r^{3}} \partial_{\alpha} r^{5} K^{\nu \mu i}+k^{2} K^{i} \\
-Q \partial_{\mu}\left(g^{\mu \nu(0)} \frac{1}{r^{3}} f_{\nu i}\right)=0 .
\end{gathered}
$$

Further operating $\epsilon_{i j k} \partial_{j}$ on the equation,

$$
\begin{gathered}
\partial_{\beta}\left[g^{\alpha \beta(0)} \frac{1}{r^{3}} \partial_{\alpha}\left(r^{5} \epsilon_{i j k} \partial_{j} K^{k}\right)\right]+\Delta_{\mathbb{R}^{3}}^{2} \epsilon_{i j k} \partial_{j} K^{k} \\
+Q \partial_{\mu}\left(g^{\mu \nu(0)} \frac{1}{r^{3}} \partial_{\nu} f_{i}\right)=0 .
\end{gathered}
$$

More explicitly,

$$
\begin{gathered}
\left(-\frac{1}{r^{3} H(r)} \partial_{t}^{2}+\partial_{r} H(r) \frac{1}{r^{3}} \partial_{r}\right)\left(r^{5} \epsilon_{i j k} \partial_{j} K^{k}+Q f_{i}\right) \\
+\Delta_{\mathbb{R}^{3}}^{2} \epsilon_{i j k} \partial_{j} K^{k}=0 .
\end{gathered}
$$

We have two sets of equations of motion (4.6) and (4.11). To simplify them, let us perform the following rescaling:

$$
r \rightarrow \frac{r_{+}}{u}, \quad t \rightarrow \frac{t}{r_{+}}, \quad \vec{y} \rightarrow \frac{\vec{x}}{r_{+}},
$$

and make the change of variables,

$$
f_{i}(r) \rightarrow \phi(r), \quad \epsilon_{i j k} \partial_{j} K^{k} \rightarrow \frac{1}{\sqrt{3} r_{+}^{2}} u^{3} \psi(r),
$$

and set $q=\frac{\mu}{\sqrt{3} r_{+}}$. The temperature $T$ is

$$
T=\frac{r_{+}}{2 \pi}\left(2-\frac{\mu^{2}}{3 r_{+}^{2}}\right) \text {. }
$$

With the rescaled variables, the Reissner-Nordström black hole is

$$
d s^{2}=\frac{1}{u^{2}}\left(-\tilde{H}(u) d t^{2}+\frac{1}{\tilde{H}(u)} d u^{2}\right)+\frac{1}{u^{2}} d \vec{x}^{2},
$$

where

$$
\tilde{H}(u)=1-\left(1+q^{2}\right) u^{4}+q^{2} u^{6} .
$$

In these coordinates, the $\mathrm{AdS}_{5}$ boundary is at $u=0$ and the black hole horizon is located at $u=1$.

Suppose that the fields $\phi$ and $\psi$ have time dependence $e^{-i \omega t}$. Then the equations of motion for the fields $\phi$ and $\psi$ give the following set of ordinary differential equations:

$$
\begin{gathered}
\frac{\omega^{2}}{\tilde{H}(u)} \phi+u \partial_{u}\left(\tilde{H}(u) u^{-1} \partial_{u} \phi\right)-k^{2} \phi \\
+8 \sqrt{3} \alpha q k u^{2} \phi-2 q u^{2} \psi=0 \\
\frac{\omega^{2}}{\tilde{H}(u)}\left(\psi-6 q u^{2} \phi\right)+u^{-1} \partial_{u}\left[\tilde{H}(u) u^{3} \partial_{u}\left(u^{-2}\left(\psi-6 q u^{2} \phi\right)\right)\right] \\
-k^{2} \psi=0 .
\end{gathered}
$$

Introducing a new function $\xi=\psi-6 q u^{2} \phi$, the equations can be written as

$$
\begin{aligned}
\frac{\omega^{2}}{\tilde{H}(u)} \phi & +u \partial_{u}\left(\tilde{H}(u) u^{-1} \partial_{u} \phi\right) \\
& -\left(k^{2}+8 \sqrt{3} \alpha q k u^{2}+12 q^{2} u^{4}\right) \phi-2 q u^{2} \xi=0 \\
\frac{\omega^{2}}{\tilde{H}(u)} \xi & +u \partial_{u}\left(\tilde{H}(u) u^{-1} \partial_{u} \xi\right)-6 q k^{2} u^{2} \phi \\
& -\left(k^{2}-8 u^{2}-9 u^{2} q^{2}+12 q^{2} u^{4}\right) \xi=0 .
\end{aligned}
$$

Interestingly, the two equations can be diagonalized by a $u$-independent matrix. That is, for some linear combinations $\phi_{1}$ and $\phi_{2}$ of $\phi$ and $\xi$, we have

$$
\frac{\omega^{2}}{\tilde{H}(u)} \phi_{i}(u)+u \partial_{u}\left(\tilde{H}(u) u^{-1} \partial_{u} \phi_{i}(u)\right)-\kappa_{i}(u) \phi_{i}(u)=0,
$$

where $i=1,2$ and

$$
\kappa_{i}(u)=k^{2}-4 \sqrt{3} \alpha k q u^{2}-2 u^{2}\left(2+q^{2}\left(2-6 u^{2}\right) \mp \sqrt{4+4 q^{4}-8 \sqrt{3} \alpha k q-8 \sqrt{3} k q^{3} \alpha+q^{2}\left(8+k^{2}\left(3+12 \alpha^{2}\right)\right)}\right),
$$

where $\kappa_{1}\left(\kappa_{2}\right)$ chooses the minus (plus) sign on the righthand side. Our numerical analysis shows that only $\phi_{2}(u)$ can be an unstable mode. It is related to the fact that, in the extremal limit, $\kappa_{2}$ corresponds to the smaller mass-squared in (3.11) in the near-horizon limit.

\section{B. Numerical analysis}

To solve the equations of motion (4.19) numerically, we impose the in-going boundary condition near the horizon $u=1$, and then evolve the solution to $u=0$, the $\operatorname{AdS}_{5}$ boundary. The asymptotic behavior of $\phi_{i}$ near $u=0$ is either $\phi_{i} \sim u^{2}$ or constant. The former is normalizable and the latter is non-normalizable. To find normalizable modes in the full Reissner-Nordström solution, we scan the initial conditions and see when the fields vanish at $u=0$.

First, let us consider the zero temperature limit $(q=\sqrt{2})$ and search for static solutions $(\omega=0)$, which signal the onset of an instability. The behavior of the fields near 
$u=1$ can be found from (4.19) as

$$
\phi_{i}=(1-u)^{-(1 / 2)+\sqrt{\left(\kappa_{i}(1)+3\right) / 12}}(1+\cdots),
$$

where terms in $\cdots$ vanish at $u=1$. In the actual numerical calculation in this section, we include several subleading terms to improve accuracy. For a given Chern-Simons coupling $\alpha$, static modes appear at discrete values of momentum $k$. The lowest two modes are plotted in Fig. 3. As mentioned before, only the second field $\phi_{2}$ has normalizable static solutions.

The two curves in Fig. 3 are denoted as I and II. Both curves terminate at $\alpha / \alpha_{\text {crit }}=1$ and $k / \mu=1.52 \cdots$. The critical value of the Chern-Simons coupling $\alpha_{\text {crit }}=$ $0.2896 \cdots$ is the one we found from the stability analysis of the near-horizon geometry in the previous section. The curves are supposed to extend over $k / \mu=1.52 \cdots$ and come back to the right in a bell-shaped curves. The upper branches of the curves represent the upper bounds of unstable modes. However, we have not been able to plot them due to inaccuracy of our numerical computation.

We also found out a static solution at zero momentum. However, for this solution, the curl of the off-diagonal metric component $\epsilon_{i j k} \partial_{j} K^{k}$ is constant on $\mathbb{R}^{3}$. This means that $K^{i}$ is linear in $\mathbb{R}^{3}$, and the solution is not normalizable.

We note that the curve II fits with the red curve which is at the lower-end of the momentum range that violates the Breitenlohner-Freedman bound in the near-horizon $\mathrm{AdS}_{2} \times \mathbb{R}^{3}$ geometry. As we saw in the previous section, the near-horizon geometry is unstable in this momentum range, thus the full Reissner-Nordström solution should also be unstable. In fact the momentum range that violates the Breitenlohner-Freedman bound is specified by $\kappa_{2}(1)<$ -3 , where $\phi_{2}(u)$ oscillates infinitely many times as they

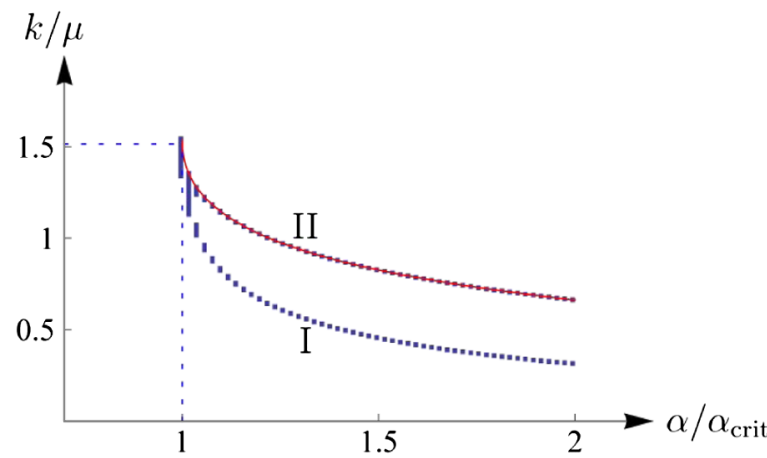

FIG. 3 (color online). For a given value of the Chern-Simons coupling $\alpha$, there is a discrete set of momenta $k$ for which static solutions exist. The curves I and II indicate two of such momenta for each $\alpha$. The red curve is the lower-end of the momentum range that violates the Breitenlohner-Freedman bound near the horizon. Note that the red curve coincides with the curve II. However, there is another curve I with a lower momentum. This means that the near-horizon analysis gives a sufficient but not necessary condition for the instability. Both curves end at the same critical value of $\alpha$. approach the horizon as can be seen from (4.21). On general ground, we expect an instability to occur in this range [34].

Interestingly, the instability condition $\kappa_{2}(1)<-3$ of the near-horizon geometry is not necessary for the instability of the full solution. This is because there is yet another curve I, located outside of this momentum range. What happens is that the curve I corresponds to a normalizable perturbation to the full Reissner-Nordström geometry, but the corresponding mode becomes non-normalizable in the near-horizon limit. Our numerical analysis shows that the critical Chern-Simons coupling $\alpha_{\text {crit }}$ for the curve I is the same as that for the curve II, even though the value of $\alpha_{\text {crit }}$ was derived from the near-horizon analysis.

To see that these static solutions indeed signal instability, let us turn on $\omega$ with positive imaginary part in (4.19). We impose the in-going boundary condition, which is

$$
\phi_{i}=e^{-(|\omega|) /(12(1-u))}(1-u)^{(7 / 36)|\omega|}(1+\cdots)
$$

in the zero temperature limit and

$$
\phi_{i}=(1-u)^{|\omega| /\left(4-2 q^{2}\right)}(1+\cdots) .
$$

at a positive temperature. Figure 4 shows the negative frequency squared as a function of the momentum $k$ at zero and finite temperature. It shows that the upper and lower curves in Fig. 3 are boundaries of unstable modes.

The occurrence of instability by the Chern-Simons coupling is summarized concisely in Fig. 1 and 2 in the introduction section of this paper. For each Chern-Simon coupling $\alpha$, Fig. 2 shows an unstable region in the momentum-temperature plane. This is related to the curve I in Fig. 3. The range of unstable momenta never includes $k=0$. The highest temperature with unstable modes is denoted as $T_{C}(\alpha)$. Figure 1 shows this critical temperature as a function of $\alpha$. Below the critical temperature $T_{C}(\alpha)$, we expect an instability, and the charge current gets a position dependent expectation value of the form of (1.1).

\section{Spontaneous current generation}

The vacuum expectation value of the current $\vec{J}$ in the dual field theory can be evaluated by extracting the asymptotic behavior of the corresponding gauge field toward the boundary of $\mathrm{AdS}_{5}$. In the absence of the Chern-Simons term, it is well-known that $\langle\vec{J}\rangle$ is given by the normalizable part of $\sqrt{-g^{(0)}} f^{r i}$ evaluated at $r \rightarrow \infty$. The normalizable mode of $f_{r i}$ decays, but that effect is compensated by the scaling behaving of the metric so that we find a finite limiting value in the low temperature phase. The ChernSimons term gives rise to an additional term of the form $\alpha \mu \epsilon_{i j k} f_{j k}$. However, it vanishes at the boundary and does not contribute to the expectation value. Thus, the vacuum expectation value of the current in the low temperature phase is given by $\sqrt{-g^{(0)}} f^{r i}$ evaluated at the boundary of 

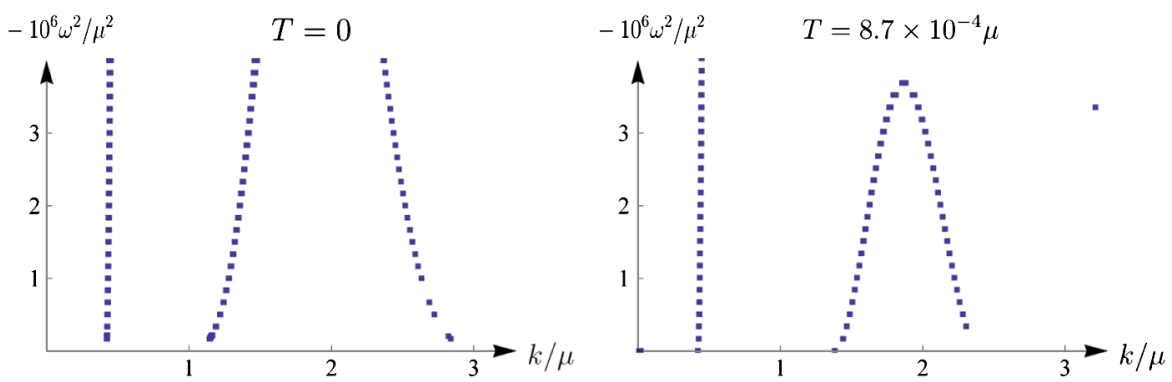

FIG. 4 (color online). Left: Negative frequency squared as a function of momentum $k$ at zero temperature when $\alpha=1.6 \alpha_{\text {crit }}$. Only positive $-\omega^{2}$ is plotted. The curves starting around 1 and 3 join to represent a tachyonic dispersion relation for the unstable mode predicted by the near-horizon analysis. The curve starting below 1 is also expected to be connected with another line in the higher momentum region to form a larger bell-shaped curve, but the large momentum part is difficult to analyze numerically. The zero momentum static solution does not extend to an unstable mode. Right: Negative frequency squared as a function of momentum at temperature $T=8.7 \times 10^{-4} \mu$.

$\mathrm{AdS}_{5}$. It takes the form

$$
\langle\vec{J}(x)\rangle=\operatorname{Re}\left(\vec{u} e^{i k x}\right),
$$

where the polarization vector $\vec{u}$ obeys

$$
\vec{k} \times \vec{u}= \pm i|k| \vec{u} .
$$

From the analysis in the previous section, it is clear that we should choose the plus (minus) sign when $\alpha E$ is positive (negative). Namely, the sign of the Chern-Simons coupling determines whether the circular polarization of the current expectation is clockwise or counter-clockwise. This configuration breaks translational and rotational symmetries, but a certain combination of the two is preserved. The polarization of the current is helical and reminds us of the cholesteric phase of liquid crystals.

Since the gauge field mixes with the metric fluctuation $h_{\mu i}$ in the bulk, the corresponding component $T_{0 i}$ of the energy-momentum tensor has a nonzero expectation value at the boundary. This is expected since the nonzero current in the spatial direction means that there is a momentum density.

\section{Spontaneous breaking of internal symmetry}

So far, we have considered the case when the gauge group in the bulk is $U(1)$. Since the $U(1)$ current commutes with itself, its expectation value does not break the $U(1)$ global symmetry on the boundary.

To realize spontaneous breaking of an internal symmetry, one possibility would be to choose the gauge group to be non-Abelian. The Chern-Simons term can be written in five dimensions if there is a symmetric tensor $d_{a b c}$ in the Lie algebra, such as in $S U(n)$ with $n \geq 3$. Suppose we turn on an electric field strength in a direction $T^{a}$ in the Lie algebra. According to $[35,36]$, the gauge kinetic term can generate instability in directions in the Lie algebra that do not commute with $T^{a}$. This breaks the symmetry homogeneously. On the other hand, the Chern-Simons term can cause a spatially modulated instability in directions where $d_{a b c} \neq 0$ with $T^{a}$. The competition of the two effects would be decided by the relative strength of the gauge coupling and the Chern-Simons coupling. It would be interesting to study such an effect in a more explicit manner to identify the gravity dual of a spatially modulated phase with spontaneous breaking of an internal symmetry.

\section{THREE-CHARGE BLACK HOLES IN TYPE IIB THEORY}

The consistent truncation of thetype IIB theory on $\mathrm{AdS}_{5} \times S^{5}$ to the $U(1)^{3}$ gauged supergravity in five dimensions was given in [37]. The bosonic action contains three gauge fields for $U(1)^{3}$ and three scalar fields $X_{1}, X_{2}, X_{3}$ subject to the constraint $X_{1} X_{2} X_{3}=1$, in addition to the metric. This low energy theory admits the three-charge black hole solutions of [38]. Here we will examine the stability of the near-horizon region of the three-charge black holes in the extremal limit.

\section{A. Case with equal charges}

Let us consider the case when the three charges are identical, which implies the scalar fields are constant $X_{1}=$ $X_{2}=X_{3}=1$. In this case, both the Lagrangian and the black hole configuration are symmetric under exchange of the three gauge fields $F_{1}, F_{2}, F_{3}$. It is convenient to take their linear combinations as

$$
\begin{aligned}
F & =\frac{1}{\sqrt{3}}\left(F_{1}+F_{2}+F_{3}\right) \\
F_{+} & =\frac{1}{\sqrt{3}}\left(F_{1}+\omega F_{2}+\omega^{2} F_{3}\right) \\
F_{-} & =\frac{1}{\sqrt{3}}\left(F_{1}+\omega^{2} F_{2}+\omega F_{3}\right) .
\end{aligned}
$$

They are eigenstates of the $\mathbb{Z}_{3}$ permutation with eigenvalues 1 and $\omega^{ \pm 1}$, where $\omega=e^{2 \pi i / 3}$. In the black hole geometry, the $\mathbb{Z}_{3}$ invariant gauge field $F$ has an electric component with $E=2 \sqrt{6}$, and $F_{ \pm}=0$. Similarly, fluctu- 
ations of the scalar fields from $X_{1}=X_{2}=X_{3}=1$ can be organized into eigenstates with eigenvalues $\omega^{ \pm 1}$ under the $\mathbb{Z}_{3}$ permutation.

The $\mathbb{Z}_{3}$ invariant sector is the minimal gauged supergravity with $\alpha=1 / 2 \sqrt{3}$. To the quadratic order, the metric and the $\mathbb{Z}_{3}$ invariant gauge field do not mix with other fields. Thus, the stability analysis with respect to them is exactly the same as the one we performed in the previous section. The three-charge black hole is barely stable in this sector, being within $0.4 \%$ of the stability bound.

Since the gauge fields $F_{+}$have zero expectation value on the black hole geometry, the $\mathbb{R}^{3}$ components of these gauge fields do not couple with other degrees of freedom in the quadratic order. It is convenient to write them as

$$
F_{ \pm}=\frac{1}{\sqrt{2}}\left(f^{(1)} \pm i f^{(2)}\right) .
$$

With the standard normalization of their kinetic terms, the Chern-Simons term takes the form

$$
\frac{1}{8 \sqrt{3}} \epsilon^{I J K L M} F_{I J}\left(a_{K}^{(1)} f_{L M}^{(1)}+a_{K}^{(2)} f_{L M}^{(2)}\right),
$$

where $a_{I}^{(i)}$ are the vector potentials for $f_{I J}^{(i)}(i=1.2)$. To the quadratic order, we can take the $\mathbb{Z}_{3}$ invariant $F_{I J}$ to be its background value $F_{I J}^{(0)}$.

Since these gauge fields do not couple to other fields in the quadratic order, their linearized equations of motion are

$$
\partial_{J}\left(\sqrt{-g} f^{(i) J I}\right)+\frac{1}{4 \sqrt{3}} \epsilon^{I J K M L} F_{J K}^{(0)} f_{L M}^{(i)}=0, \quad(a=1,2) .
$$

Comparing this with (2.4), we find $\alpha= \pm 1 / 4 \sqrt{3}$. Since $E= \pm 2 \sqrt{6}$ as in the previous example, the mass squared is given by

$$
-4 \alpha^{2} E^{2}=-2
$$

It is greater than the Breitenlohner-Freedman bound $\left(m_{\mathrm{BF}}^{2}=-3\right.$ in our unit), and quadratic fluctuations in these gauge fields are stable.

\section{B. Case with nonequal charges}

Next, let us consider the case when the three charges are different. The five-dimensional Lagrangian is derived in [37]

$$
\begin{aligned}
16 \pi G_{5} \mathcal{L}= & \sqrt{-g}\left(R-\frac{1}{2}\left(\partial \phi_{1}\right)^{2}-\frac{1}{2}\left(\partial \phi_{2}\right)^{2}+4 \sum_{a} X_{a}^{-1}\right. \\
& \left.-\frac{1}{4} \sum_{a} X_{a}^{-2}\left(F^{a}\right)^{2}\right)+\frac{1}{4} \epsilon^{I J K L M} F_{I J}^{1} F_{K L}^{2} A_{M}^{3} .
\end{aligned}
$$

$X_{a}$ are functions of the two scalars $\phi_{1}$ and $\phi_{2}$ subject to the constraint $X_{1} X_{2} X_{3}=1$. The Lagrangian admits $\mathrm{AdS}_{5}$ black holes parametrized by three charges $q_{1}, q_{2}$, and $q_{3}$.
The metric is given by

$$
\begin{aligned}
d s^{2}= & -\left(H_{1} H_{2} H_{3}\right)^{-(2 / 3)} h(r) d t^{2}+\left(H_{1} H_{2} H_{3}\right)^{1 / 3} \\
& \times\left(\frac{d r^{2}}{h(r)}+r^{2} d \Omega_{3}^{2}\right) \\
H_{a}(r)= & 1+\frac{q_{a}}{r^{2}}, \quad q_{a}=\mu \sinh ^{2} \beta_{a}, \quad a=1,2,3 \\
X_{a}= & H_{a}^{-1}\left(H_{1} H_{2} H_{3}\right)^{1 / 3} \quad h(r)=1-\frac{\mu}{r^{2}}+r^{2} H_{1} H_{2} H_{3} \\
A_{a}= & \left(1-H_{a}^{-1}\right) \operatorname{coth} \beta_{a} d t .
\end{aligned}
$$

This is the metric whose foliating transverse space is $S^{3}$. If it is $\mathbb{R}^{3}$ instead, the $S^{3}$ metric $d \Omega_{3}^{2}$ is replaced by the flat metric and $h(r)$ and $A^{a}$ are replaced with

$$
h(r)=-\frac{\mu}{r^{2}}+r^{2} H_{1} H_{2} H_{3} \quad A_{a}=\frac{1-H_{a}^{-1}}{\sinh \beta_{a}} d t .
$$

Given the charges $q_{a}$, it may be possible to choose $\mu$ such that the black hole becomes extremal. That is, the inner and the outer horizons coincide. For the extremal case, the near-horizon geometry is $\mathrm{AdS}_{2} \times S^{3}$ or $\mathrm{AdS}_{2} \times \mathbb{R}^{3}$ : if the horizon occurs at $r=r_{0}$, for small $\rho=r-r_{0}, h(r)=$ $\frac{1}{2} h^{\prime \prime}\left(r_{0}\right) \rho^{2}$. Hence, the geometry becomes

$$
d s^{2}=\frac{1}{a_{1}}\left[-\rho^{2} d t^{2}+\frac{d \rho^{2}}{\rho^{2}}\right]+\frac{1}{a_{2}} d \Omega_{3}^{2},
$$

where $\quad a_{1}=\frac{1}{2}\left(H_{1} H_{2} H_{3}\right)^{-(1 / 3)} h^{\prime \prime}, \quad$ and $\quad a_{2}=$ $\left(H_{1} H_{2} H_{3}\right)^{-(1 / 3)} r_{0}^{-2}$ for $S^{3}$ and $a_{2}^{-1} d \Omega_{3}^{2}$ is replaced with the flat metric for $\mathbb{R}^{3}$. $H_{a}$ and $h^{\prime \prime}$ are implicitly evaluated at $r=r_{0}$.

We want to analyze the linear fluctuations near the horizon in the extremal limit. Let $F_{a}=F_{a}^{(0)}+f_{a}=$ $F_{a}^{(0)}+d a_{a}$ and $g_{I J}=g^{(0)}+h_{I J}$. If we focus on the fluctuations of the $a_{i}$ and $h_{\mu i}$ fields only, we find that the linear fluctuations of the scalar fields $\phi_{1}$ and $\phi_{2}$ do not couple to them. Therefore, we may use the background value of the scalar fields. In this case, we can derive the equations of motion as in the previous case, and the result is

$$
\begin{array}{r}
\left(\Delta_{2}+\Delta_{3}\right) f^{1}-X_{1}^{2} E_{3} d^{*} f^{2}-X_{1}^{2} E_{2} d^{*} f^{3}+E_{1} d K=0 \\
-X_{2}^{2} E_{3} d^{*} f^{1}+\left(\Delta_{2}+\Delta_{3}\right) f^{2}-X_{2}^{2} E_{1} d^{*} f^{3}+E_{2} d K=0 \\
-X_{3}^{2} E_{2} d^{*} f^{1}-X_{3}^{2} E_{1} d^{*} f^{2}+\left(\Delta_{2}+\Delta_{3}\right) f^{3}+E_{3} d K=0 \\
\frac{E_{1}}{X_{1}^{2}} \Delta_{2}{ }^{*} f^{1}+\frac{E_{2}}{X_{2}^{2}} \Delta_{2}^{*} f^{2}+\frac{E_{3}}{X_{3}^{2}} \Delta_{2}{ }^{*} f^{3}+\left(\Delta_{2}+\Delta_{3}+4 a_{2}\right) d K=0 .
\end{array}
$$

$E_{a}$ are the electric fields such that $d A_{a}=$ $E_{a}\left(H_{1} H_{2} H_{3}\right)^{-(1 / 6)} d t \wedge d r$. The above four equations give a mass matrix equation 
$\operatorname{det}\left(\begin{array}{cccc}m^{2}-k^{2} & -E_{3} X_{1}^{2} k & -E_{2} X_{1}^{2} k & E_{1} \\ -E_{3} X_{2}^{2} k & m^{2}-k^{2} & -E_{1} X_{2}^{2} k & E_{2} \\ -E_{2} X_{3}^{2} k & -E_{1} X_{3}^{2} k & m^{2}-k^{2} & E_{3} \\ \frac{E_{1}}{X_{1}^{2}} m^{2} & \frac{E_{2}}{X_{2}^{2}} m^{2} & \frac{E_{3}}{X_{3}^{2}} m^{2} & m^{2}-k^{2}+4 a_{2}\end{array}\right)=0$.

Solving this equation for $m^{2}$, we obtain the mass spectrum.

When two of the three charges are the same, we can analyze the mass spectrum analytically for the $\mathrm{AdS}_{2} \times \mathbb{R}^{3}$ geometry. In this case, only the ratio of the charges matter. Let the charge assignments be $\left(q_{a}\right)=(1, q, q)$. Demanding $f\left(r_{0}\right)=f^{\prime}\left(r_{0}\right)=0$ at some $r=r_{0}$, we obtain the relation $q=x(2 x+1)$ and $\mu=4 x(1+x)^{3}$ where $x=r_{0}^{2}$. Let us parametrize the extremal solutions in terms of $x$. Then the various functions at the horizon are given by

$$
\begin{gathered}
H_{1}=x^{-1}(1+x), \quad H_{2}=H_{3}=2(1+x), \\
H_{1} H_{2} H_{3}=4 x^{-1}(1+x)^{3}, \\
X_{1}=2^{2 / 3} x^{2 / 3}, \quad X_{2}=X_{3}=2^{-(1 / 3)} x^{-(1 / 3)}, \\
E_{1}=2^{7 / 3} x^{5 / 6}, \quad E_{2}=E_{3}=2^{1 / 3} x^{-(2 / 3)}(1+2 x)^{1 / 2},
\end{gathered}
$$

$a_{1}=2^{4 / 3} x^{-(2 / 3)}(1+4 x), \quad \Lambda=-2^{1 / 3} x^{-(2 / 3)}(1+4 x)$.

When the two charges are the same, there is a $\mathbb{Z}_{2}$ symmetry exchanging the two charges. Since the gravity is insensitive to this exchange, only the combination $f^{2}+f^{3}$ couples to the metric component and $f^{2}-f^{3}$ decouples. The decoupled mode is analyzed by considering the mass matrix in (5.10) with the eigenvector $(0,1,-1,0)$ for some $m^{2}$. Because of the fact that $E_{2}=E_{3}$ and $X_{2}=X_{3}$, the only condition that we need to satisfy is

$$
m^{2}-k^{2}+E_{1} X_{2}^{2} k=0 .
$$

Therefore, $m^{2}$ has the minimum value when $k=\frac{E_{1} X_{2}^{2}}{2}$, in which case $m^{2}=-\frac{E_{1}^{2} X_{2}^{4}}{4}=-2^{4 / 3} x^{1 / 3}$. The BreitenlohnerFreedman bound is $-\frac{a_{1}}{4}=-2^{-(2 / 3)} x^{-(2 / 3)}(1+4 x)$. Their ratio is $\frac{4 x}{1+4 x}$, which is always lower than 1 . That is, the mass squared is always above the bound.

Of course, it is possible that there are other modes that go below the bound. But this turns out not to be the case. To see this, let us evaluate the determinant (5.10) when the mass-squared $m^{2}$ takes the value $-\frac{1}{4} a_{1}$, which is the Breitenlohner-Freedman bound. Then this is a function of $k$ and $x$. We can check that this function is always positive, meaning that the roots of the determinant equation, which are the possible values of the mass-squared, are all greater than the Breitenlohner-Freedman bound.

When all three charges are different, we have not been able to solve the equations analytically, so we resorted to a numerical method. Given three charges, we first adjust the parameter $\mu$ in (5.6) so that it gives an extremal black hole. Then we evaluate the metric and the functions at the horizon and solve the mass matrix Eq. (5.10) for $m^{2}$. In both $\mathrm{AdS}_{2} \times S^{3}$ or $\mathrm{AdS}_{2} \times \mathbb{R}^{3}$, however, no unstable modes are found for a large range of the three charges. The bound is always barely satisfied.

In this section, we studied stability of the three-charge black hole in the near-horizon limit. As we saw in the previous section, the near-horizon analysis gives a sufficient but not necessary condition for the instability at $T=$ 0 . However, the critical value of the Chern-Simons coupling is given correctly from the near-horizon analysis. Thus, we expect that our conclusion in this section would not be modified even if we perform the analysis in the full black hole geometry.

\section{ACKNOWLEDGMENTS}

We thank M. Fisher, K. Hashimoto, G. Horowitz, S. Kinoshita, A. Kitaev, M. Kitazawa, H. Liu, K. Murata, M. Oshikawa, Y. Tachikawa, and T. Takayanagi for discussions. H. O. and C.-S. P. are supported in part by DOE Grant No. DE-FG03-92-ER40701. H. O. is also supported in part by the World Premier International Research Center Initiative of MEXT of Japan and by a Grant-in-Aid for Scientific Research (C) under Grant No. 20540256 of JSPS. S.N. is supported by the Grant-in-Aid for the Global COE Program "The Next Generation of Physics, Spun from Universality and Emergence" of MEXT of Japan. S. N. was also supported by the SRC Program of the KOSEF through the Center for Quantum Space-time of Sogang University under Grant No. R11-2005-021 and by the YST program of Asia Pacific Center for Theoretical Physics at the initial stage of the present work. H. O. thanks the Albert Einstein Institute in Golm, the Aspen Center for Physics, the Galileo Galilei Institute in Florence, the Kavli Institute for Theoretical Physics in Santa Barbara, and the Yukawa Institute for Theoretical Physics in Kyoto for their hospitality.
[1] S. A. Hartnoll, Classical Quantum Gravity 26, 224002 (2009).

[2] C. P. Herzog, J. Phys. A 42, 343001 (2009).
[3] S. Deser, R. Jackiw, and S. Templeton, Phys. Rev. Lett. 48, 975 (1982).

[4] S. Deser, R. Jackiw, and S. Templeton, Ann. Phys. (N.Y.) 
140, 372 (1982); 185, 406(E) (1988); 281, 409 (2000);

[5] S. K. Domokos and J.A. Harvey, Phys. Rev. Lett. 99, 141602 (2007).

[6] A. Chamblin, R. Emparan, C. V. Johnson, and R. C. Myers, Phys. Rev. D 60, 064018 (1999).

[7] M. Cvetic and S.S. Gubser, J. High Energy Phys. 04 (1999) 024.

[8] S. J. Rey, Prog. Theor. Phys. Suppl. 177, 128 (2009).

[9] P. Fulde and R. A. Ferrell, Phys. Rev. 135, A550 (1964).

[10] A. I. Larkin and Y. N. Ovchinnikov, Zh. Eksp. Teor. Fiz. 47, 1136 (1964) [Sov. Phys. JETP 20, 762 (1965)].

[11] M. G. Alford, J. A. Bowers, and K. Rajagopal, Phys. Rev. D 63, 074016 (2001).

[12] D. V. Deryagin, D. Y. Grigoriev, and V. A. Rubakov, Int. J. Mod. Phys. A 7, 659 (1992).

[13] E. Shuster and D. T. Son, Nucl. Phys. B573, 434 (2000).

[14] S. A. Brazovskii, Sov. Phys. JETP, 41, 85 (1975).

[15] See, for example, P. C. Hohenberg and J. B. Swift, Phys. Rev. E 52, 1828 (1995), and the references therein.

[16] E. Witten, Adv. Theor. Math. Phys. 2, 253 (1998).

[17] S. Benvenuti, L. A. Pando Zayas and Y. Tachikawa, Adv. Theor. Math. Phys. 10, 395 (2006).

[18] C. Vafa, arXiv:hep-th/0509212.

[19] H. Ooguri and C. Vafa, Nucl. Phys. B766, 21 (2007).

[20] V. V. Klimov, Yad. Fiz. 33, 1734 (1981) [Sov. J. Nucl. Phys. 33, 934 (1981)].

[21] H. A. Weldon, Phys. Rev. D 26, 2789 (1982).

[22] E. Braaten, R. D. Pisarski, and T. C. Yuan, Phys. Rev. Lett. 64, 2242 (1990).
[23] For review, see, for example, M. Le Bellac, Thermal Field Theory, Cambridge Monographs on Mathematical Physics (Cambridge University Press, Cambridge, UK, 2000).

[24] J. P. Gauntlett, R. C. Myers, and P. K. Townsend, Classical Quantum Gravity 16, 1 (1999).

[25] J. Kunz and F. Navarro-Lerida, Phys. Rev. Lett. 96, 081101 (2006).

[26] J. Erdmenger, M. Haack, M. Kaminski, and A. Yarom, J. High Energy Phys. 01 (2009) 055.

[27] N. Banerjee, J. Bhattacharya, S. Bhattacharyya, S. Dutta, R. Loganayagam, and P. Surowka, arXiv:0809.2596.

[28] M. Torabian and H. U. Yee, J. High Energy Phys. 08 (2009) 020.

[29] D. T. Son and P. Surowka, Phys. Rev. Lett. 103, 191601 (2009).

[30] Y. Matsuo, S. J. Sin, S. Takeuchi, and T. Tsukioka, arXiv:0910.3722.

[31] B. Sahoo and H.-Y. Yee, arXiv:0910.5915.

[32] M. Gunaydin, G. Sierra, and P. K. Townsend, Nucl. Phys. B242, 244 (1984).

[33] A. Fujii and R. Kemmoku, Phys. Lett. B 459, 137 (1999).

[34] G. Gibbons and S. A. Hartnoll, Phys. Rev. D 66, 064024 (2002).

[35] S. S. Gubser, Phys. Rev. Lett. 101, 191601 (2008).

[36] S. S. Gubser and S.S. Pufu, J. High Energy Phys. 11 (2008) 033.

[37] M. Cvetic et al., Nucl. Phys. B558, 96 (1999).

[38] K. Behrndt, M. Cvetic, and W. A. Sabra, Nucl. Phys. B553, 317 (1999). 